\title{
Gamma-Interferon Inhibits Collagen Synthesis In Vivo in the Mouse
}

\author{
Richard D. Granstein," George F. Murphy," Randall J. Margolis," Michael H. Byme," and Edward P. Amento" \\ *Wellman Laboratory, Department of Dermatology, and ${ }^{\S}$ Arthritis Unit, Department of Medicine, Massachusetts General Hospital \\ and Harvard Medical School, Boston, Massachusetts 02114; and ${ }^{\ddagger}$ Dermatopathology Laboratory, Brigham and Women's \\ Hospital and Harvard Medical School, Boston, Massachusetts 02115
}

\begin{abstract}
Subcutaneous implantation of osmotic pumps into $\mathrm{CAF}_{1}$ mice resulted in the formation of thick fibrous capsules around the pumps. When pumps were loaded with recombinant murine gamma-interferon (rMuIFN- $\gamma$ ) to deliver $2 \times 10^{3} \mathrm{U} / \mathrm{h}$ for $14 \mathrm{~d}$, there was a marked decrease in thickness and collagen content of the capsules from rMuIFN- $\gamma$-treated animals compared with capsules from animals receiving diluent alone. The collagen content of the capsules was estimated by hydroxyproline analysis of the tissue and by quantitative electron microscopy of collagen bundles. Heat-inactivated rMuIFN- $\gamma$ failed to reduce the fibrotic response in this assay. These results provide compelling evidence that gamma-interferon can down-regulate collagen synthesis in vivo and suggest the possibility that this lymphokine may be useful in the treatment of disease states characterized by excessive fibrosis.
\end{abstract}

\section{Introduction}

Collagen, as the major fibrous protein in connective tissue, is the primary structural component of the organs and tissues in the human body. The amount of collagen in tissues is maintained by control of the balance between synthesis and degradation of collagen and is transiently altered in repair processes such as wound healing (1). Abnormalities in collagen turnover may lead to pathological fibrosis of the skin in diseases such as scleroderma $(2)$, keloid formation $(3,4)$, or pathological fibrosis of internal organs such as the liver or lungs. Studies from several laboratories have demonstrated that products of inflammatory cells may function in the regulation of collagen synthesis and degradation (5), particularly in cell culture systems (6-13). The monocyte product, interleukin 1 (IL-1), increases collagen and fibronectin synthesis by dermal and synovial fibroblasts and chondrocytes $(13,14)$ and murine mammary epithelial cells $(15)$. The lymphokine, gamma-interferon (IFN- $\gamma$ ), ${ }^{1}$ in contrast, inhibits types I and III collagen and fibronectin synthesis by dermal and sy-

This work was presented in part at the combined Association of American Physicians/American Society for Clinical Investigation/American Federation for Clinical Research meeting, May 1986, in Washington, DC and was published in abstract form (1984. Clin. Res. 34:617A).

Address reprint requests to Dr. Granstein, Department of Dermatology, Massachusetts General Hospital, Wellman 2, 50 Blossom Street, Boston, MA 02114.

Received for publication 18 September 1986.

1. Abbreviations used in this paper: IFN- $\gamma$, gamma-interferon; rMuIFN$\gamma$, recombinant murine gamma-interferon.

J. Clin. Invest.

(C) The American Society for Clinical Investigation, Inc. $0021-9738 / 87 / 04 / 1254 / 05 \quad \$ 1.00$

Volume 79, April 1987, 1254-1258 novial fibroblasts and type II collagen by chondrocytes in a dosedependent manner (16-18). The decrease in collagen synthesis is associated with decreased levels of cellular messenger RNA (mRNA) for these proteins (19-21).

IFN- $\gamma$ has been shown to influence the function of a variety of cells in vitro and in vivo. This lymphokine alters the phenotype of macrophages, several effector functions such as the increased expression of Fc receptors and enhanced killing of tumor cells, and increased macrophage $\mathrm{H}_{2} \mathrm{O}_{2}$-releasing capacity and antiprotozoal activity (22-25). The expression of the major histocompatibility complex class II antigens on many normal and tumor cells is also enhanced or induced by IFN- $\gamma$ both in vitro and in vivo $(17,26,27)$. In order to examine the physiological relevance of our observations on collagen synthesis, an in vivo model has been developed to assess the potential modulation by IFN- $\gamma$ of collagen synthesis in the fibrotic reaction of a murine host to an implanted foreign body.

In studies of the actions of several different drugs, osmotic pumps (28) have been implanted subcutaneously or intraperitoneally to deliver soluble substances into rats or mice. We noted that the subcutaneous implantation of these pumps into mice is followed by the formation of a thick fibrous capsule in 1-2 wk. We took advantage of this model of new connective tissue matrix formation to study the in vivo influence of recombinant murine IFN- $\gamma(\mathrm{rMuIFN}-\gamma)$ on connective tissue remodeling. We reasoned that the influence of rMuIFN- $\gamma$ could be more readily seen with the elicitation of new fibrous tissue formation than by observation of the normal process of connective tissue remodeling.

\section{Methods}

Mice. Female $\mathrm{CAF}_{1}(\mathrm{BALB} / \mathrm{c} \times \mathrm{A} / \mathrm{J})$ mice were obtained from the Jackson Laboratory (Bar Harbor, ME). The animals were 8-14 wk old at the start of each experiment and the age of the animals did not vary by $>2$ wk within an experiment. The mice had free access to Purina Mouse Chow (Ralston Purina Co., St. Louis, MO) and chlorinated water and were housed in a facility where ambient light was regulated on a 12-h light/dark cycle.

$I F N-\gamma$. Biologically active rMuIFN- $\gamma$ (specific activity, $10.3 \times 10^{6}$ $\mathrm{U} / \mathrm{mg}$ ) was kindly provided by Dr. H. M. Shepard (Genentech, Inc., South San Francisco, CA), and was stored at $4^{\circ} \mathrm{C}$ in concentrated form and diluted immediately before use. Units of activity were determined by a cytopathic effect inhibition assay using L929 murine fibroblasts challenged with encephalomyocarditis virus. Titers were expressed in international units on the basis of the murine IFN- $\alpha / \beta$ research reference standard G002-904-511. This preparation contained $<0.25 \mathrm{EU} / \mathrm{ml}$ by the Limulus amebocyte lysate test where $1 \mathrm{EU}$ is the amount of Limulus amebocyte lysate-reactive material in $2 \mathrm{ng}$ of U. S. Pharmacopeia reference standard endotoxin (Escherichia coli lipopolysaccharide).

Pump implantation. Small osmotic pumps were obtained from Alza Corp. (model 2002, Palo Alto, CA). These cylindrical pumps, when loaded and assembled, measure $3.0 \mathrm{~cm}$ along the long axis and $0.7 \mathrm{~cm}$ in diameter and deliver $0.5 \mu \mathrm{l} / \mathrm{h}$ for $14 \mathrm{~d}$. They have an outer membrane of cellulose ester and have a cap at the exit port composed of ethylene copolymer. 
The pumps are lucent for a portion of the distance from the exit port to the distal end of the pump (Fig. 1). Pumps were loaded with rMuIFN$\gamma$ in phosphate-buffered saline containing $0.1 \%$ mouse serum or medium alone. A small, full-thickness cut was made on the caudal side of the dorsum of ether-anesthetized mice and a subcutaneous pouch prepared by blunt dissection. A pump was then placed in the pouch with the exit port at the cranial end of the pouch. Each mouse received only one pump. The skin was closed with one or two autoclips (Becton, Dickinson \& Co., Parsippany, NJ).

Fibrous capsules. Fibrous capsules were noted to form around pumps in days to weeks after implantation. At indicated times, animals were killed and an area containing a pump excised en bloc. Capsules were then carefully dissected off of the pumps.

Transmission electron microscopy. Small specimens of capsule were minced into cubes $\sim 1 \mathrm{~mm}^{3}$, fixed in Karnovsky's II solution for $5 \mathrm{~h}$, and rinsed in $0.1 \mathrm{M}$ sodium cacodylate buffer ( $\mathrm{pH} 7.4$ ) for $2 \mathrm{~h}$. Postfixation was achieved with $2 \%$ osmium tetroxide for $2 \mathrm{~h}$. After two, 15-min rinses with $0.1 \mathrm{M}$ sodium cacodylate buffer, the tissue was dehydrated in graded ethanol solutions and embedded in an Epon-Araldite mixture. Ultrathin sections subsequently cut for electron microscopy were stained with uranyl acetate and lead citrate and examined with a JEOL JEM 100s electron microscope (JEOL USA, Peabody, MA). Computerized image analysis of randomly selected cellular fields was performed as previously described $(29,30)$.

Hydroxyproline content determination. Tissue was hydrolyzed in 5 $\mathrm{ml}$ of $6 \mathrm{~N} \mathrm{HCl}$ and dried, and hydroxyproline content was determined as previously described (31).

Statistical analysis. The significance of differences in hydroxyproline content between groups was assessed by the Student's $t$ test. All experiments were performed at least twice.

\section{Results}

Capsule formation. Initial studies were designed to compare capsule formation surrounding pumps containing rMuIFN- $\gamma$ $\left(6.7 \times 10^{5} \mathrm{U} / \mathrm{ml}\right)$ with control pumps containing medium alone. These pumps were loaded to deliver $2 \times 10^{3} \mathrm{U} / \mathrm{h}$ of rMuIFN$\gamma .7 \mathrm{~d}$ after implantation of pumps into each group of three CAF mice, the area of the pumps was excised en bloc and the

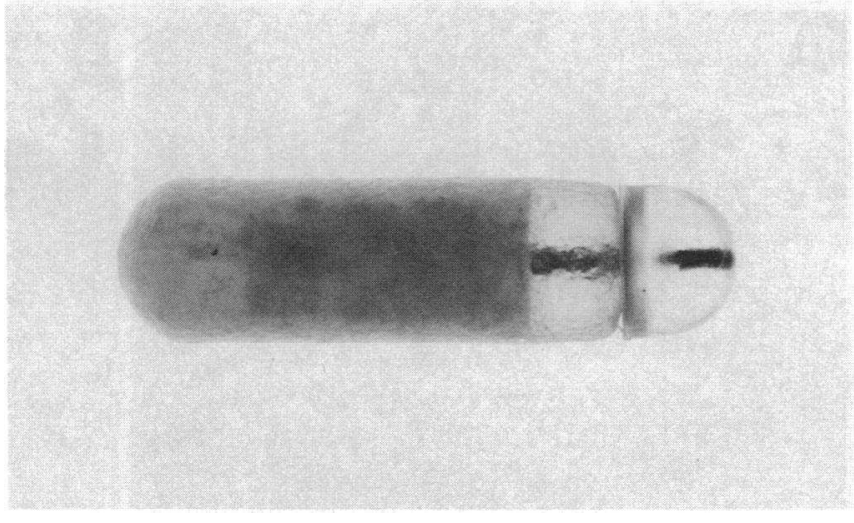

Figure 1. Fully assembled osmotic pump with exit port on the right. Note the lucent area of the pump (right).

capsule was examined. At this time point, no grossly observable capsule was present around the pumps loaded with rMuIFN- $\gamma$ although some thin strands of tissue did adhere to the pumps. In contrast, the control pumps had readily observable capsules.

In a second experiment, pumps loaded with rMuIFN- $\gamma$ or medium alone were implanted into each of three $\mathrm{CAF}_{1}$ mice and were not disturbed for $14 \mathrm{~d}$. At $14 \mathrm{~d}$, the animals were killed and the capsules surrounding the pumps were carefully removed. Capsules surrounding pumps containing rMuIFN- $\gamma$ appeared to be thinner and smaller compared with control capsules. Fig. 2 compares the gross appearance of a capsule dissected from a pump delivering rMuIFN- $\gamma$ with that from around a control group.

In an additional experiment, pumps loaded with media alone or media containing rMuIFN- $\gamma$ were implanted into $\mathrm{CAF}_{1}$ mice and the mice were weighed at $0,5,10$, and $15 \mathrm{~d}$. No significant differences in weight were noted between the groups at any time point, and no individual mouse varied in weight by $>5 \%$ between any time points in either group. In all experiments, mice

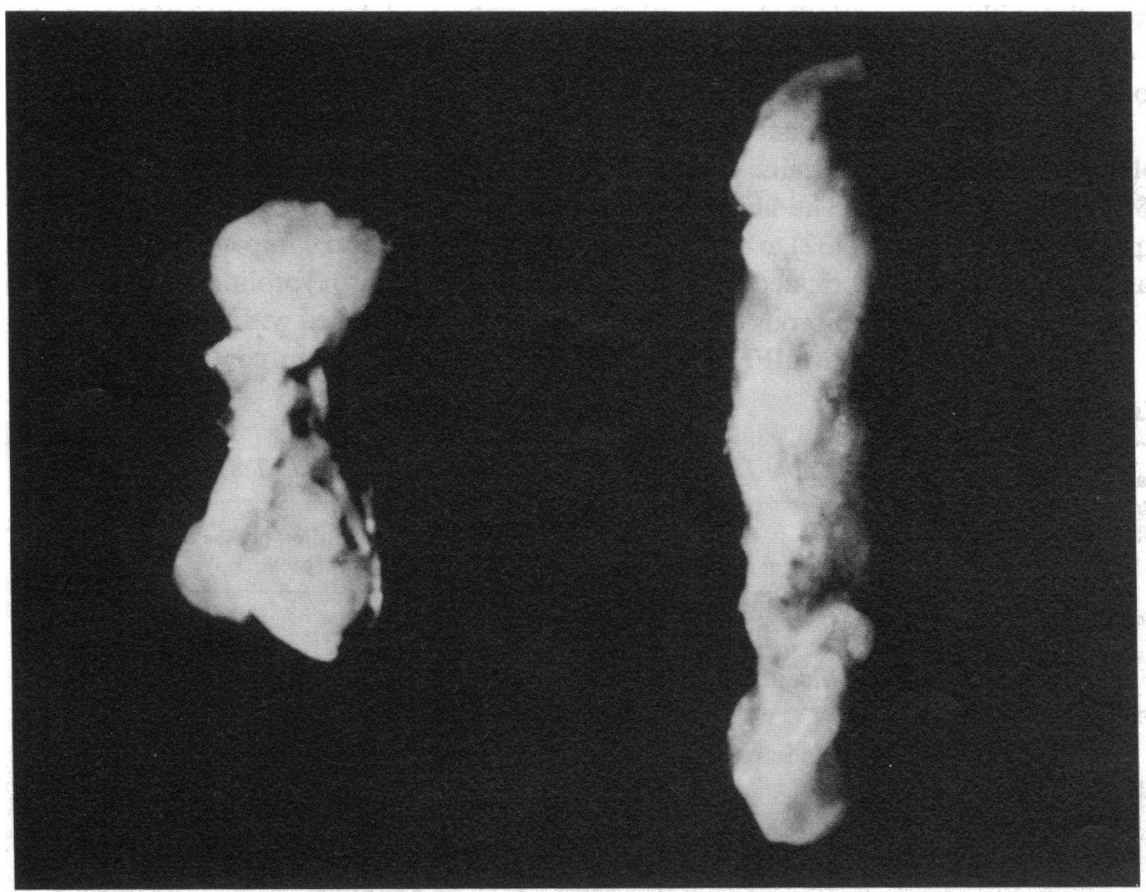

Figure 2. Capsules were removed from a pump containing rMuIFN- $\gamma($ left $)$, and one containing media alone (right). 


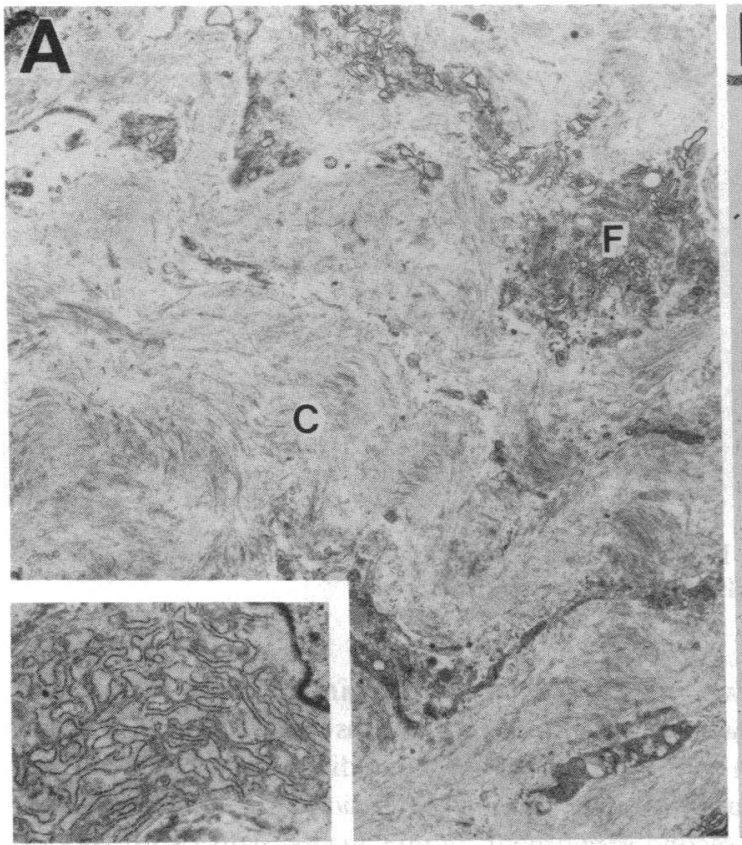

Figure 3. Transmission electron micrographs of capsules from a pump containing medium alone $(A)$ or containing rMuIFN- $\gamma(B)$ after $14 \mathrm{~d}$. In the control specimen, fibroblasts $(F)$ contain dilated endoplasmic reticulum (inset); the extracellular matrix is composed predominantly

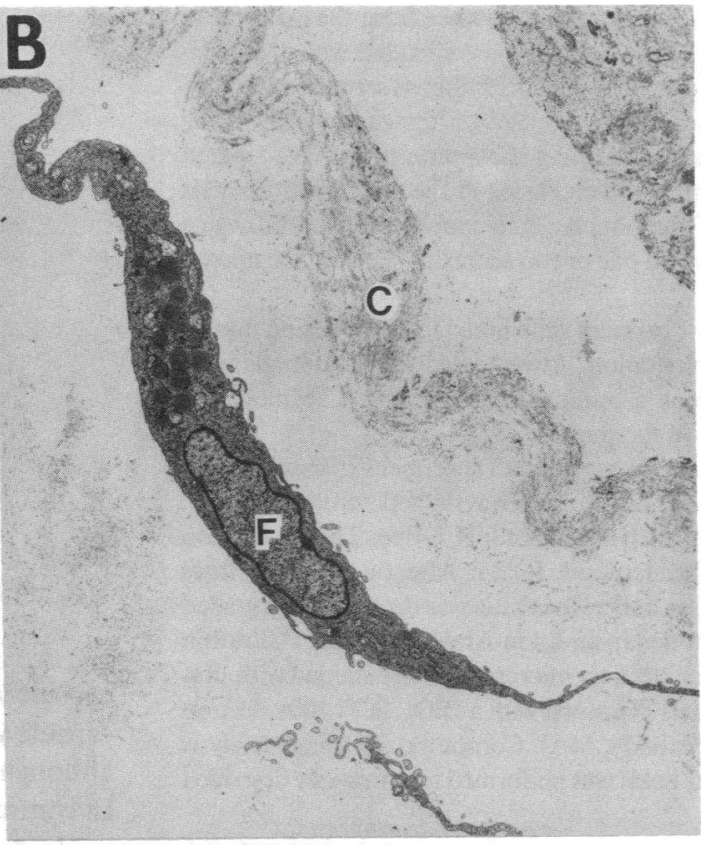

of closely aggregated mature collagen bundles $(C)$. In the rMuIFN- $\gamma-$ treated specimens, fibroblasts appear inactive and strands of loosely packed collagen are observed within an electron-lucent background $(A, \times 3,000$; inset, $\times 10,000 ; B, \times 3,000)$.

Hydroxyproline content. Specimens were obtained by dissecting the one-quarter of the capsule close to and surrounding the exit port of each pump. This was done easily by utilizing a landmark present on each pump. These specimens were assayed for hydroxyproline content (31). As shown in Table I, the control specimens had much greater hydroxyproline content than the rMuIFN- $\gamma$-treated specimens. Even when normalized to dry weight, a greater collagen content was found in the control specimens compared with specimens from rMuIFN- $\boldsymbol{\gamma}$-treated animals. Normalizing to sample weight minimizes the difference in hydroxyproline content observed, because much of the weight of specimens from either group is due to collagen content. Marked differences were also seen when the collagen content of an entire capsule was analyzed (control of $955 \mu \mathrm{g}$ of hydroxyproline compared to $205 \mathrm{mg}$ of hydroxyproline for rMuIFN- $\gamma$ ). The ability of heat-inactivated $\left(80^{\circ} \mathrm{C}\right.$ for $\left.30 \mathrm{~min}\right) \mathrm{rMuIFN}-\gamma$ (32) to affect collagen formation was also compared with controls treated with medium alone. The hydroxyproline content of the fibrous capsules surrounding pumps filled with heat-inactivated rMuIFN- $\gamma$ did not vary significantly from controls implanted

Table II. Hydroxyproline Content of Capsule Tissue

\begin{tabular}{lll}
\hline Content of pumps* & $\begin{array}{l}\text { Mean hydroxyproline content } \\
\text { per 1/4 capsule }\end{array}$ & $P$ value \\
\hline & $\mu g \pm S E M$ & \\
Medium plus heat-treated IFN- $\gamma$ & $187 \pm 14$ & 0.964 \\
Medium alone & $235 \pm 21$ & \\
\hline
\end{tabular}

* rMuIFN- $\gamma$ was subjected to a temperature of $80^{\circ} \mathrm{C}$ for $30 \mathrm{~min}$. Three specimens were analyzed in each group.
Mean

hydroxyproline content per mg

Content
pumps* content per $1 / 4$ capsule

$\mu g / m g \pm S E M$

Medium plus rMuIFN- $\gamma$

$$
51.3 \pm 18.4
$$

$5.4 \pm 0.8$
* Three specimens were analyzed in each group.

$14.8 \pm 1.7$

Medium alone $\quad 395.0 \pm 40.0 \quad 14.8 \pm 1.7 \quad<.002 \quad<0$


with pumps containing media alone at $14 \mathrm{~d}$ (Table II); thus, the capacity of rMuIFN- $\gamma$ to affect collagen synthesis is heat-labile.

\section{Discussion}

The presence of various inflammatory and immunocompetent cells including B and T lymphocytes and monocyte/macrophages in tissues undergoing pathologic fibrosis or healing processes involving fibrosis (33-36) has suggested that lymphokines and monokines might play a role in the regulation of fibrous tissue production. As noted above, IL-1 has been found to increase collagen and fibronectin synthesis in vitro $(13,14)$ and we have recently shown that 10 daily subcutaneous injections of epidermal cell-derived thymocyte activating factor (similar or identical to IL-1) into mice results in subcutaneous fibrosis in addition to producing an inflammatory response (37). Also as noted above, in vitro studies have shown that IFN- $\gamma$ down-regulates collagen and fibronectin synthesis (16-18) and this decreased synthesis is associated with decreased levels of mRNA (19-21). Fibrous capsule formation around alloplastic implants in humans and animals, including breast implants $(38,39)$, silicone plates (40), Marlex mesh (41), and nylon velour pouches (42) has been observed previously. Our finding that a similar phenomenon occurs with the osmotic pumps allowed us to develop an in vivo model for soluble mediator effects on new collagen synthesis. We show with the data presented in the current study that IFN- $\gamma$ can down-regulate collagen synthesis in vivo. The demonstration that this activity is heat-labile correlates with the previously described loss of biological activity by heat treatment of IFN- $\gamma$ (32). The sensitivity of IFN- $\gamma$ to heat also permits evaluation of the potential role of endotoxin in these results. Any endotoxin possibly contaminating the rMuIFN- $\gamma$ could not account for these findings inasmuch as endotoxin is not affected by heating to $80^{\circ} \mathrm{C}$ for $30 \mathrm{~min}(43)$.

These findings support a physiological role for IFN- $\gamma$ in matrix turnover, and suggest that activated $\mathrm{T}$ lymphocytes in inflammatory lesions may be capable of decreasing the local synthesis of collagen. The mechanism by which collagen synthesis is inhibited by IFN- $\gamma$ cannot be assessed in this type of in vivo experiment, although it presumably reflects the inhibition of collagen mRNA synthesis seen in in vitro studies (19-21). More importantly, the finding of in vivo activity by rMuIFN- $\gamma$ in down-regulating collagen synthesis suggests a possible therapeutic role for this lymphokine.

Numerous disease states, including keloids, hypertrophic scars, scleroderma, morphea, graft vs. host disease, and idiopathic pulmonary fibrosis, among many others, are characterized in part by local or systemic pathologic fibrosis. One may speculate that the pathogenesis of some of these disorders may be due, in part, to abnormal regulation or response to IFN- $\gamma$ or other modulators with similar biological activities. If adverse side effects prove to be acceptable, IFN- $\gamma$ might be useful in the therapy of some of these diseases.

\section{Acknowledgments}

We thank Dr. Stephen M. Krane (Massachusetts General Hospital and Harvard Medical School) for helpful suggestions and review of the manuscript, Ms. Eileen Fonferko for technical assistance, and Ms. Diane Dalton for preparation of this manuscript.

This work was supported by National Institutes of Health grant AM03564 and a grant from the Arthur O. Gullan M. Wellman Foundation. Dr. Granstein is the recipient of National Institutes of Health Clinical
Investigator Award AM-01425-02; Dr. Amento is the recipient of an Arthritis Foundation Investigator Award.

\section{References}

1. Pinnell, S. R. 1981. Regulation of collagen synthesis. J. Invest. Dermatol. 79(Suppl. 1):73-76.

2. Fleischmajer, R., J. J. Perlish, and M. Duncan. 1983. Scleroderma: a model for fibrosis. Arch. Dermatol. 119:957-962.

3. Murray, J. C., S. V. Pollack, and S. R. Pinell. 1981. Keloids: a review. J. Am. Acad. Dermatol. 4:461-470.

4. Chvapil, M., and C. F. Koopman, Jr. 1984. Scar formation: physiology and pathological states. Otolaryngol. Clin. North Am. 17:265272.

5. Evered, D., and J. Whelan, editors. 1985. Fibrosis. Ciba Found. Symp. 114:1-250.

6. Jiminez, S. A., W. McArthur, and J. Rosenbloom. 1979. Inhibition of collagen synthesis by mononuclear cell supernatants. J. Exp. Med. 150:1421-1431.

7. McArthur, W., K. Derr, M. Dixon, S. A. Jiminez, and J. Rosenbloom. 1982. Immune modulation of connective tissue functions: studies on the production of collagen synthesis inhibitory factor by populations of human peripheral blood mononuclear cells. Cell Immunol. 74:126139.

8. Schmidt, J. A., S. B. Mizel, D. Cohen, and I. Green. Interleukin I, a potential regulator of fibroblast proliferation. J. Immunol. 128:21772182.

9. Wahl, S. M., and C. L. Gately. 1982. Modulation of fibroblast growth by a lymphokine of human $\mathrm{T}$ cell and continuous $\mathrm{T}$ cell line origin. J. Immunol. 130:1226-1230.

10. Postlethwaite, A. E., G. N. Smith, C. L. Mainarch, J. M. Seyer, and A. H. Kang. 1984. Lymphocyte modulation of fibroblast function in vitro: stimulation and inhibition of collagen production by different effector molecules. J. Immunol. 132:2470-2477.

11. Duncan, M. R., J. S. Perlish, and R. Fleischmajer. 1984. Lymphokine/monokine inhibition of fibroblast proliferation and collagen production: role in progressive systemic sclerosis (PSS). J. Invest. Dermatol. 83:377-384.

12. Korotzer, T. I., R. C. Page, G. A. Granger, and P. S. Rabinovich. 1982. Regulation of growth of human diploid fibroblasts by factors elaborated by activated lymphoid cells. J. Cell Physiol. 111:247-254.

13. Krane, S. M., J.-M. Dayer, L. S. Simon, and M. S. Byrne. 1985. Mononuclear cell-conditioned medium containing mononuclear cell factor (MCF), homologous with interleukin I, stimulates collagen and fibronectin synthesis by adherent synovial cells: effects of prostaglandin $\mathrm{E}_{2}$ and indomenthacin. Collagen Relat. Res. 5:99-117.

14. Goldring, M. B., and S. M. Krane. 1986. Modulation of collagen synthesis in human chondrocyte cultures by interleukin I. J. Bone Miner. Res. 1:56(Suppl. 1).

15. Matsushima, K., M. Bano, W. R. Kidewell, and J. J. Oppenheim. 1985. Interleukin I increases collagen type IV production by murine mammary epithelial cells. J. Immunol. 134:904-909.

16. Jiminez, S. A., B. Freundlich, and J. Rosenbloom. 1984. Selective inhibition of human diploid fibroblast collagen synthesis by interferons. J. Clin. Invest. 74:1112-1116.

17. Amento, E. P., A. K. Bhan, K. G. McCullogh, and S. M. Krane. 1985. Influence of gamma interferon on synovial fibroblastlike cells: Ia induction and inhibition of collagen synthesis. J. Clin. Invest. 76:836848.

18. Duncan, M. R., and B. Berman. 1985. Gamma interferon is the lymphokine and beta interferon the monokine responsible for inhibition of fibroblast collagen production and late but not early fibroblast proliferation. J. Exp. Med. 162:516-527.

19. Rosenbloom, J., G. Feldman, B. Freundlich, and S. A. Jiminez. 1984. Transcriptional control of human diploid fibroblast collagen synthesis by gamma-interferon. Biochem. Biophys. Res. Commun. 123:365372.

20. Stephenson, M. L., S. M. Krane, E. P. Amento, P. A. McCroskery, 
and M. Byrne. 1985. Immune interferon inhibits collagen synthesis by rheumatoid synovial cells associated with decreased levels of the procollagen mRNAs. FEBS (Fed. Eur. Biochem. Soc.) Lett. 180:43-50.

21. Goldring, M. B., L. J. Sandell, M. L. Stephenson, and S. M. Krane. 1986. Immune interferon suppresses levels of procollagen mRNA and type II collagen synthesis in cultured human articular and costal chondrytes. J. Biol. Chem. 261:9049-9056.

22. Perussia, B., E. T. Dayton, V. Fanning, P. Thiagarajnn, J. Hoxie, and G. Trinchieri. 1983. Immune interferon and leukocyte-conditioned medium induce normal and leukemic myeloid cells to differentiate along the monocytic pathway. J. Exp. Med. 148:2058-2080.

23. Guyre, P. M., P. M. Marganelli, and R. Miller. 1983. Recombinant immune interferon increases immunoglobulin G Fc receptors on cultured human mononuclear phagocytes. J. Clin. Invest. 72:393-397.

24. Le, J., W. Prensky, Y. K. Yip, Z. Cheng, T. Hoffman, H. C. Stevenson, I. Balazs, J. R. Sadlik, and J. Vilcek. 1983. Activation of human monocyte cytotoxicity by natural and recombinant immune interferon. J. Immunol. 131:2821-2826.

25. Murray, H. W., G. L. Spitalny, and C. F. Nathan. 1985. Activation of mouse peritoneal macrophages in vitro and in vivo by gamma interferon. J. Immunol. 134:1619-1622.

26. Kelley, V. E., W. Fiers, and T. B. Strom. 1984. Cloned human interferon- $\gamma$ but not interferon- $\alpha$ or $\beta$ induces expression of HLA-DR determinants by fetal monocytes and myeloid leukemic cell lines. $J$. Immunol. 132:240-245.

27. Skoskiewicz, M. J., R. B. Colvin, E. E. Schneeberger, and P. S. Russell. 1985. Widespread and selective induction of major histocompatability complex-determined antigens in vivo by $\gamma$-interferon. J. Exp. Med. 162:1645-1664.

28. Theeuwes, F., and S. I. Yum. 1976. Principles of the design and operation of generic osmotic pumps for the delivery of semisolid or liquid drug formulations. Ann. Biomed. Eng. 4:343-353.

29. Murphy, G. F., T. J. Harrist, A. K. Bhan, and M. C. Mihm, Jr. 1983. Distribution of cells surface antigens in histiocytosis $X$ cells. Quantitative immunoelectron microscopy using monoclonal antibodies. Lab. Invest. 48:90-97.

30. Jones, M. H., and T. Okagaki. 1986. Technologic progress in digital (computerized) imaging applicable to medicine. Inf. Pathol. 1: 33-37.
31. Bergman, I., and R. Loxley. 1963. Two improved and simplified methods for the spectrophotometric determination of hydroxyproline. Anal. Chem. 35:1961-1965.

32. Schultz, R. M., and M. A. Chirigos. 1978. Similarities among factors that render macrophages tumoricidal in lymphokine and interferon preparations. Cancer Res. 38:1003-1007.

33. Gisslow, M. T., and B. C. McBride. 1975. A rapid sensitive collagenase assay. Arch. Biochem. 68:70-78.

34. Leibovich, S. J., and R. Ross. 1975 . The role of the macrophage in wound repair. Am. J. Pathol. 78:71-100.

35. Crystal, R. G., J. D. Fullmer, W. C. Roberts, M. L. Moss, B. R Line, and H. Y. Reynolds. 1978. Idiopathic pulmonary fibrosis. Clinical, histological, radiographic, physiologic, scintigraphic, cytologic, and biochemical aspect. Ann. Intern. Med. 85:769-788.

36. Fleischmajer, R., J. S. Perlish, and W. P. West. 1977. Ultrastructure of cutaneous cellular infiltrates in scleroderma. Arch. Dermatol. 113:1661-1666.

37. Granstein, R. D., R. J. Margolis, S. B. Mizel, and D. N. Sauder. 1986. In vivo inflammatory activity of epidermal cell-derived thymocyte activating factor and recombinent interleukin 1 in the mouse. J. Clin. Invest. 77:1020-1027.

38. Smahel, J. 1977. Histology of the capsules causing constrictive fibrosis around breast implants. Br. J. Plast. Surg. 30:324-329.

39. McCoy, B. J., P. Person, and I. K. Cohen. 1984. Collagen production and types in fibrous capsules around breast implants. Plast. Reconstr. Surg. 73:924-927.

40. Abla, A. A., J. C. Maroon, J. S. Kennerdell, and Z. L. Deed. 1985. Fibrosis surrounding a silicone implant simulating recurrent orbital hemangiomas. J. Neurosurg. 63:467-469.

41. Elliott, M. P., and G. L. Juler. 1979. Comparison of Marlex mesh and microporous Teflon sheets when used for hernia repair in the experimental animal. Am. J. Surg. 137:342-344.

42. McCutcheon, E. P., J. M. Evans, and F. H. Wibel. 1982. A fabric pouch for maintaining transducer and catheter terminations. J. Biomed. Mater. Res. 16:105-115.

43. Elin, R. J., and S. M. Wolfe. 1973. Bacterial endotoxin. In CRC Handbook of Microbiology. Vol. II. A. I. Laskin and H. A. Lechevalier, editors. CRC Press, Boca Raton, FL. 215-237. 